\title{
The Effect of Acute Rhodiola rosea Ingestion on Exercise Heart Rate, Substrate Utilisation, Mood State, and Perceptions of Exertion, Arousal, and Pleasure/Displeasure in Active Men
}

\author{
Michael J. Duncan ${ }^{1,2}$ and Neil D. Clarke ${ }^{1}$ \\ ${ }^{1}$ Department of Biomolecular and Sports Sciences, Coventry University, Coventry CV1 5HB, UK \\ ${ }^{2}$ Human Performance Laboratory, Department of Biomolecular and Sports Sciences, Coventry University, James Starley Building, \\ Priory Street, Coventry CV 5HB, UK \\ Correspondence should be addressed to Michael J. Duncan; michael.duncan@coventry.ac.uk
}

Received 24 February 2014; Revised 29 March 2014; Accepted 2 April 2014; Published 27 April 2014

Academic Editor: Laura Guidetti

\begin{abstract}
Copyright (C) 2014 M. J. Duncan and N. D. Clarke. This is an open access article distributed under the Creative Commons Attribution License, which permits unrestricted use, distribution, and reproduction in any medium, provided the original work is properly cited.
\end{abstract}

\begin{abstract}
The aim of this study was to examine the effect of acute Rhodiola rosea ( $R$. rosea) ingestion on substrate utilisation, mood state, RPE, and exercise affect. Ten males (mean age \pm S.D. $=26 \pm 6$ years) completed two 30 -minute cycling trials at an intensity of $70 \%$ of $\dot{\mathrm{VO}}_{2 \max }$ following ingestion of either $3 \mathrm{mg} \cdot \mathrm{kg}^{-1}$ body mass of $R$. rosea or placebo using a double-blind, crossover design. During exercise, heart rate and RPE were recorded. Participants completed measures of mood state and exercise affect before and after exercise. Expired air samples were taken during exercise to determine substrate utilisation. Repeated measures analysis of variance indicated that RPE was significantly lower at 30 minutes into exercise versus placebo $(P=0.003)$. Perceptions of arousal $(P=0.05)$ and pleasure were significantly higher after exercise with $R$. rosea compared to placebo $(P=0.003)$. Mood state scores for vigor were also higher in $R$. rosea condition compared to placebo $(P=0.008)$. There were no significant differences in energy expenditure, carbohydrate, or fat oxidation between conditions $(P>0.05)$. Ingestion of $R$. rosea favourably influenced RPE and exercise affect without changes in energy expenditure or substrate utilization during 30-minute submaximal cycling performance.
\end{abstract}

\section{Introduction}

Rhodiola rosea ( $R$. rosea) is an herb that grows in mountainous regions of North America, Europe, and Asia. It has been used in traditional folk medicine for centuries as a treatment for fatigue and mood disorders [1]. $R$. rosea has been extensively studied by scientists in the former Soviet Union and has predominantly been found to result in favourable effects on exercise performance [2]. However, this prior work has been lacking in experimental control [3]. Some recent studies have supported the results of earlier work by identifying antioxidant and anti-inflammatory properties of $R$. rosea $[3,4]$. Another work has suggested ingestion of $R$. rosea appears to be effective, either acutely [5] or with daily supplementation [6], for reducing perceived fatigue and improving cognition $[5,7]$, as well as reducing markers of physiological and psychological stress $[7,8]$.
Although $R$. rosea ingestion has been identified as a means to reduce physical fatigue, its efficacy during exercise is unclear. Animal based research has shown increased swim time to exhaustion in rats $[3,9]$ but the impact of $R$. rosea ingestion on exercise performance in humans is equivocal. Some studies have shown no effect of $R$. rosea ingestion on exercise performance $[4,10-12]$ whilst others have supported its use $[13,14]$. The array of protocols and supplementation doses used in prior studies has also limited comparisons between studies and provides one potential explanation for the discrepancy in their findings. For example, de Bock et al. [13] reported that an acute dose of $R$. rosea increased time to exhaustion during an incremental cycle ergometer protocol. Conversely, in the same study, 4 weeks of $R$. rosea supplementation did not significantly improve cycling performance. This was the first study to show an acute impact of $R$. rosea ingestion on human exercise performance. More 
recent research by Noreen et al. [14] examined the efficacy of a $3 \mathrm{mg} \cdot \mathrm{kg}^{-1}$ body mass dose of $R$. rosea on 6 -mile cycle time trial performance in 18 active women. They reported that $R$. rosea significantly decreased submaximal exercise heart rate, reduced RPE, and improved time trial performance time. Noreen et al. [14] subsequently suggested that acute $R$. rosea ingestion might be employed by active individuals while exercising to complete a set distance in the shortest time.

Such data are interesting; as if $R$. rosea does positively impact on exercise, it may be safe and legal way to enhance exercise performance in athletes and the exercise experience in recreational gym users. The mechanism by which $R$. rosea might improve performance is also unclear. Abidov et al. [15] demonstrated that an extract of $R$. rosea significantly prolonged the duration of exhaustive swimming in rats and stimulated ATP synthesis in muscle during exercise. These findings led Perfumi and Mattioli [16] to speculate that, in addition to enhancing the catecholaminergic system, the ability of $R$. rosea to increase performance involves an improvement in cellular energy metabolism. However, some studies have suggested that $R$. rosea acts to acutely increase endogenous opioid production or receptor sensitivity [2, 13] which subsequently impacts on brain dopamine and serotonin and cardiac activity and attenuates the perception of effort at a given workload [14]. However, as only two studies have examined acute $R$. rosea ingestion on exercise performance to date, further data is needed on this topic. Moreover, both de Bock et al. [13] and Noreen et al. [14] employed performance based protocols to examine the effect of $R$. rosea ingestion. While these are valuable in understanding the effect of a nutritional supplement on performance responses, different protocols may be better placed to evidence any effect of $R$. rosea on exercise performance. For example, using steady state submaximal exercise in the presence of $R$. rosea and placebo would allow for power output to remain constant across a given period. This then allows for better like-forlike comparison of data between the active supplement and placebo trials. Moreover, when employing protocols where intensity is modified throughout (as is the case with both time trials and time to exhaustion) understanding any impact of the exercise protocol of substance ingested becomes more difficult because even small increases in exercise intensity have been shown to markedly change feelings of pleasure during exercise [17].

Furthermore, given the postulation that $R$. rosea acts through increased opioid production, it is perhaps surprising that few studies have examined its impact on affect. Noreen et al. [14] reported no effect of $R$. rosea ingestion on mood state, as assessed using the POMS questionnaire. However, changes in mood state with exercise have been reported following ingestion of a range of substances including caffeine $[18,19]$ and carbohydrate [20]. Hedonic theory [21] postulates that hedonic responses (i.e., pleasure versus displeasure) following a behaviour influence decisions regarding whether or not to repeat that behaviour. As a consequence if nutritional manipulation enhances the pleasure response to exercise, according to hedonic theory, it may mean individuals are more likely to exercise [21]. This is important as different exercise intensities and individual fitness will create variability in the affective response to exercise [22-24] and the effect of nutritional manipulation on these responses is not well known. Changes in mood and feelings of pleasure may be one reason why individuals do not participate in regular exercise and physical activity [19] and favourable affective states benefit exercise performance [25]. Research by DaSilva et al. [26] has also identified that affective and perceptual responses to exercise differ depending on adiposity status. Assuming that the affective response to exercise is the same in recreational exercises compared to high level performers may therefore lead to erroneous conclusions regarding the exercise-affect relationship. Thus, while the extant literature examining the effect of $R$. rosea ingestion has focused on performance measures, the current study seeks to develop this work by employing a model that can be applied to recreational exercisers and is more applicable to the exercising public in general.

The aim of this study was to examine the effect of acute $R$. rosea ingestion on substrate utilisation, mood state, RPE, perceptions of arousal, and pleasure/displeasure in a population of recreationally active men. It was hypothesized that ingestion of $R$. rosea would positively influence perceived exertion during exercise as well as enhancing mood and perceptions of arousal and pleasure/displeasure before and after cycling exercise. Testing these hypotheses will address gaps in knowledge on the effect of $R$. rosea by examining their effects on an active but not performance oriented group, more typical of regularly active individuals in the general population. The study will also combine physiological and psychological measures to address gaps in the literature base by assessing substrate utilisation, heart rate, RPE, mood state, and perceptions of arousal and pleasure/displeasure to provide a multidisciplinary investigation of the effect of $R$. rosea during exercise.

\section{Method}

2.1. Design. This study employed a within-participants double-blind cross-over design whereby participants visited the laboratory on 3 occasions at the same time of day in a well-rested and well-hydrated state. Ten male, recreational exercisers (mean age \pm S.D. $=26 \pm 6$ years) participated in this study. All participants were asked to refrain from vigorous exercise and maintain normal dietary patterns in the 48 hours prior to testing and were asked not to consume caffeine for 24 hours before testing. During the first visit participants completed a familiarization session with the measures to be used in the subsequent experimental trials and completed an incremental exercise test to assess $\dot{\mathrm{V}}_{2}$ max . Inclusion criteria included being male and habitually engaged in recreational physical activity of more than 3 but less than 10 hours per week and not including formal competitive sports performance. Participants were excluded if they had a musculoskeletal or cardiovascular contraindication to exercise, were taking any medication that could impact mood/affect, engaged in less than 3 or more than 10 hours physical activity per week, or were engaged in competitive sports activity as part of their habitual physical activity. 
TABLE 1: Descriptive information (mean \pm S.D.) of participants' height, body mass, resting heart rate, and maximal oxygen uptake.

\begin{tabular}{lccccccc}
\hline & Height $(\mathrm{m})$ & \multicolumn{2}{c}{ Mass $(\mathrm{kg})$} & \multicolumn{2}{c}{ Resting heart rate $(\mathrm{bpm})$} & \multicolumn{2}{c}{$\dot{\mathrm{VO}}_{2 \mathrm{max}}\left(\mathrm{mL}^{2} \mathrm{~kg}^{-1} \cdot \mathrm{min}^{-1}\right)$} \\
$\mathrm{M}$ & S.D. & $\mathrm{M}$ & S.D. & M & S.D. & M & S.D. \\
\hline 1.72 & 0.03 & 67.7 & 6.3 & 79.8 & 17.3 & 50.5 & 6.6 \\
\hline
\end{tabular}

The incremental exercise test was performed on a mechanically braked cycle ergometer (Monark Exercise AB, Sweden) to assess $\dot{\mathrm{VO}_{2} \text { max }}$ following previously published guidelines [27]. Initially, participants were fitted to the ergometer and performed a 5 min warm-up at 25 Watts. The initial work rate for the test was 80 Watts with the work rate increasing by 40 Watts every 3 minutes. This protocol, using 3-minute stages rather than a ramp protocol, was employed based on prior research suggesting that this form of protocol provides more reliable physiological measures, particular for the population characteristics examined in this study (i.e., active but not cycling trained specifically) [27]. Participants were asked to maintain their pedal cadence at $70 \mathrm{rpm}$ and were given visual feedback from the Monark control box in order to do this. Expired air was collected via the Douglas bag technique during the final minute of each incremental exercise stage. Samples were analyzed for oxygen and carbon dioxide content (Servomex, Crowborough, England) and expired air volume (Harvard dry gas meter, Harvard Apparatus, Kent, England) with values for oxygen consumption $\left(\dot{\mathrm{VO}}_{2}\right)$ and carbon dioxide production $\left(\dot{\mathrm{V}} \mathrm{CO}_{2}\right)$ subsequently calculated. Heart rate (Polar Electro, Kempele, Finland) and rating of perceived exertion (RPE), using the Borg 6-20 RPE scale [28], were recorded during the final 15 seconds of each workload. Exercise was continued to exhaustion in order for measurement of $\dot{\mathrm{VO}}_{2 \text { max }}$ to be made. Participants were judged to have reached $\dot{\mathrm{VO}}_{2 \text { max }}$ if they presented at least 3 of the following: (a) a respiratory exchange ratio of greater than 1.1, (b) a heart rate during the last stage of testing that was \pm 10 beats of age predicted maximum heart rate, (c) an RPE of 18 or greater, (d) a plateau in $\mathrm{VO}_{2}$ with an increase in workload, and (e) volitional fatigue. These criteria were employed based on those commonly used for maximal oxygen uptake [29]. All participants met these criteria during their incremental exercise test. Mean \pm S.D. of participants' baseline $\dot{\mathrm{VO}}_{2 \text { max }}$ values was $50.5 \pm 6.6 \mathrm{~mL} \cdot \mathrm{kg}^{-1} \cdot \mathrm{min}^{-1}$ (range: $\left.38.8-58.4 \mathrm{~mL} \cdot \mathrm{kg}^{-1} \cdot \mathrm{min}^{-1}\right)$. Descriptive information for the participants is presented in Table 1 .

2.2. Experimental Protocol. On completion of the $\dot{\mathrm{VO}}_{2 \max }$ testing and following a period of at least 72 hours participants completed two 30-minute submaximal cycling trials at a workload of $70 \% \dot{\mathrm{VO}}_{2 \max }$ in a fasted state. Self-report of dietary intake was employed to assess and control dietary intake in the 24 hours prior to exercise trials. This was used to ensure that the same/similar per testing meals were consumed prior to experimental trials and that caffeine and alcohol had not been consumed in the 24 hours prior to testing. The duration and intensity of exercise were chosen as it sits within the American College of Sports Medicine guidelines for the prescription of exercise for health benefit [30]. On a molecular basis, the main active ingredients of $R$. rosea appear to be Tyrosol and its glucoside known as salidroside and following oral ingestion of $100 \mathrm{mg} \cdot \mathrm{kg}^{-1}$ salidroside, the half-life of salidroside appears to be $1.32 \pm$ $0.22 \mathrm{~h} \mathrm{[31]}$. Therefore, conditions were randomised, separated by 48-72 hours, and consisted of a $R$. rosea condition where $3 \mathrm{mg} \cdot \mathrm{kg}^{-1}$ body mass of $R$. rosea (Indigo Herbs, Glastonbury, UK) placed in a coloured, opaque gelatin capsule or a placebo $\left(3 \mathrm{mg} \cdot \mathrm{kg}^{-1}\right.$ body mass of maltodextrin, (MyProtein, Northwich, UK)) was ingested with $250 \mathrm{~mL}$ water. The capsules were indistinguishable between $R$. rosea and placebo conditions and were ingested $60 \mathrm{~min}$ before exercise on an empty stomach. The amount of total maltodextrin ingested was approximately $170 \mathrm{mg}$ and thus highly unlikely to have had any impact on exercise performance or metabolism [14]. The dose of $R$. rosea used was based on the previous work of de Bock et al. [13] and Noreen et al. [14] who observed improvements in endurance performance following acute ingestion of $R$. rosea.

During each of the cycling trials, heart rate was monitored (Polar RS400, Polar Electro Oy, Kempele, Finland) continuously and assessed every 10 minutes. Participants were also asked to provide ratings of perceived exertion (RPE) using the Borg 6-20 scale [28]. Prior to commencing the experimental trials the memory-anchoring approach [32] was employed whereby participants were asked to remember a time when they were at rest (attributed to RPE of 6) and a time when they had reached a level of exertion that was maximal attributed to RPE of 20. The RPE scale was presented to students for 1 minute prior to their rating of perceived exertion at each time point and was not visible other than this. Prior to any substance ingestion, $60 \mathrm{~min}$ after ingestion (at the onset of each exercise bout) and immediately on completion of each exercise bout, participants also completed the feeling scale (FS) as a measure of pleasure and displeasure [33]. This is an 11-item single-item scale ranging from +5 (very good) to -5 (very bad) that is used to quantify pleasure/displeasure. The Felt Arousal Scale (FAS) was also used as a measure of arousal [34]. This is a six-item scale ranging from 1 (low arousal) to 6 (high arousal). Mood state was also assessed using the fatigue and vigour subscales of the Brunel Mood State Inventory (BRUMS) [35]. This is a well-established, reliable, and valid measure of mood state that has been previously employed to assess the mood state response to various exercise modes $[18,35,36]$. The fatigue and vigor subscales were chosen in particular as prior research $[1,7]$ has suggested $R$. rosea may particularly influence feelings of fatigue and/or vigor.

Participants were introduced to these scales during their first visit to the laboratory (prior to establishment of $\dot{\mathrm{VO}}_{2 \text { max }}$ ). Standardised instructions for completing the FS and FAS were read to participants at the beginning of each trial. 


\section{Substrate Oxidation}

Prior to cycling (but whilst seated on the exercise bike) at $14 \mathrm{~min}$ and $29 \mathrm{~min}$ during each exercise bout expired air samples were collected using the Douglas bag technique to estimate fat and carbohydrate $(\mathrm{CHO})$ oxidation rates. The collection time was $60 \mathrm{~s}$ and samples were analysed for oxygen and carbon dioxide content (Servomex, Crowborough, England) that was calibrated at two points before each assessment using gases of known concentrations $\left(15.12 \% \mathrm{O}_{2}, 5.1 \% \mathrm{CO}_{2}\right)$ in accordance with manufacturers guidelines and expired air volume (Harvard dry gas meter, Harvard Apparatus, Kent, England) with values for oxygen consumption $\left(\dot{\mathrm{VO}}_{2}\right)$ and carbon dioxide production $\left(\dot{\mathrm{VCO}}_{2}\right)$ subsequently calculated. Energy expenditure $\left(\mathrm{Kcal} \cdot \mathrm{min}^{-1}\right)$ and the oxidation rates for carbohydrate and fat $\left(\mathrm{g} \cdot \mathrm{min}^{-1}\right)$ were then calculated according to the Weir [37] and Frayn [38] equations, respectively.

3.1. Statistical Analysis. Data were analysed in a number of ways. A series of 2 (substance ingested) $\times 4$ (time point) ways repeated measures analysis of variance was used to examine any differences in heart rate and RPE. A series of 2 (substance ingested) X 3 (time point, before ingestion, after ingestion but before exercise, and after exercise) ways repeated measures analysis of variance was used to examine any differences in mood state and perceptions of arousal and pleasure/displeasure and substrate utilisation. Where any significant differences were discovered, Bonferroni pairwise multiple comparisons were used to determine where the differences lay. Partial $\eta^{2}$ was used as a measure of effect size, statistical significance was set at $P=0.05$ a priori, and the Statistical Package for Social Sciences (Version 20) was used for all analysis (SPSS Inc., IL, USA).

\section{Results}

In respect to heart rate, results indicated no significant interactions or main effect due to the substances ingested. There was however a significant main effect for time ( $F$ 3, $27=266.7, P=0.0001, P \eta^{2}=0.967$ ) where heart rate was significantly increased throughout the exercise bout irrespective of substance ingested. Mean \pm SE of heart rate (bpm) was $77 \pm 4$ at rest compared to $151 \pm 4,158 \pm 4$, and $162 \pm 4$ at 10,20, and 30 minutes into the exercise bout, respectively. Bonferroni comparisons indicated that heart rate was significantly different at all time points $(P=0.001$ in all cases).

In the case of RPE, results indicated a significant substance $\times$ time interaction $\left(F 3,27=6.12, P=0.003, P \eta^{2}=\right.$ 0.405 , see Figure 1). RPE data were not significantly different at rest and at 10 and 20 minutes into the exercise bout between the two conditions. However, RPE in the placebo condition was significantly higher than that in the $R$. rosea condition 30 minutes into the exercise bout $(P=0.003)$.

Scores from the fatigue subscale of the BRUMS indicated a significant main effect across time $(F 2,18=12.879, P=$ $\left.0.0001, P \eta^{2}=0.589\right)$ where scores were significantly higher

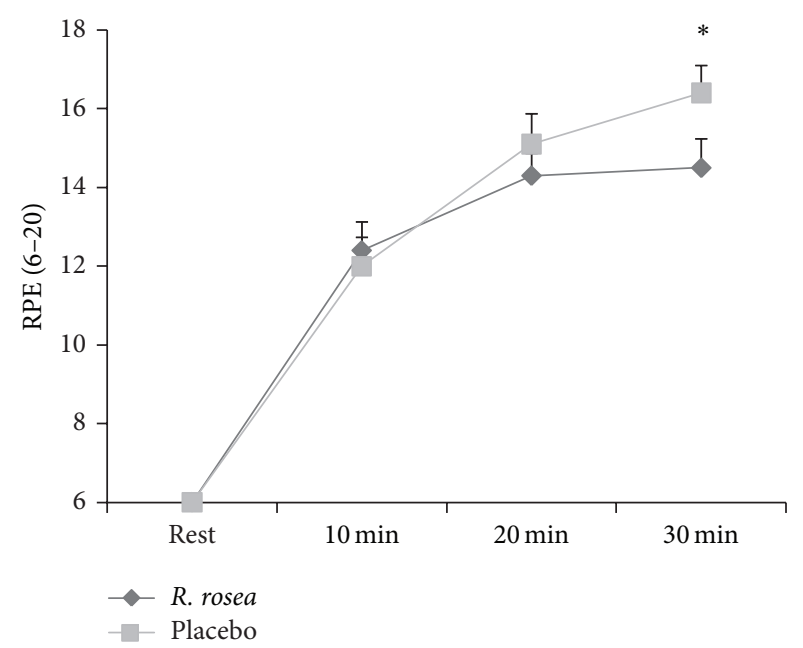

Figure 1: Mean \pm SE of RPE (6-20) at rest and during 30 min submaximal cycling between $R$. rosea and placebo conditions $\left({ }^{*} \mathrm{P}=\right.$ $0.003)$.

after exercise compared to before exercise $(P=0.028)$ and compared to before exercise but after ingestion $(P=0.008)$. There were no other significant main effects of interactions for fatigue subscale data. In regard to the vigor subscale of the BRUMS, there was no significant substance $\times$ time interaction. There were however significant main effects for time $\left(F 2,18=4.435, P=0.027, P \eta^{2}=0.350\right)$ and for the substance ingested $\left(F 2,9=11.692, P=0.008, P \eta^{2}=0.565\right)$. Scores for vigor were significantly lower at after exercise compared to after ingestion but before exercise $(P=0.04)$. Mean \pm SE for vigor scores was $6.9 \pm 0.56$ before ingestion, $7.4 \pm 0.44$ after ingestion but before exercise, and $5.6 \pm 0.74$ after exercise. For the substance ingested, scores for vigor were significantly higher in the $R$. rosea condition $(P=0.008)$ compared to the placebo. Mean \pm SE for vigor was $7.6 \pm 0.52$ in the $R$. rosea condition compared to $5.7 \pm 0.57$ in the placebo condition.

Perceptions of arousal were also significantly different in the presence of $R$. rosea compared to placebo $(F 1,9=5.0$, $\left.P=0.05, P \eta^{2}=0.357\right)$ with scores for arousal being higher $(3.1 \pm 0.19)$ in the presence of $R$. rosea compared to placebo $(2.8 \pm 0.11)$. When data for feeling of pleasure/displeasure were assessed, there was however a significant condition $\times$ time effect $\left(F\right.$ 2, $18=12.795, P=0.0001, P \eta^{2}=0.587$, see Figure 2). This followed a similar pattern to RPE with no significant difference evident before ingestion $(P=0.591)$ or after ingestion but before exercise $(P=0.08)$. There was however significantly lower scores for pleasure after exercise in the placebo condition compared to the $R$. rosea condition $(P=0.003)$.

Energy expenditure was significantly higher during exercise $\left(F 2,18=130.921, P<0.001, P \eta^{2}=0.936\right)$ (see Figure 3$)$, irrespective of the trial $\left(F 1,9=1.198, P=0.302, P \eta^{2}=0.117\right)$. The rate of total carbohydrate oxidation (Figure 4 ) was not significantly different between trials $(F 1,9=0.317, P=0.587$, $\left.P \eta^{2}=0.034\right)$. Carbohydrate oxidation remained relatively 


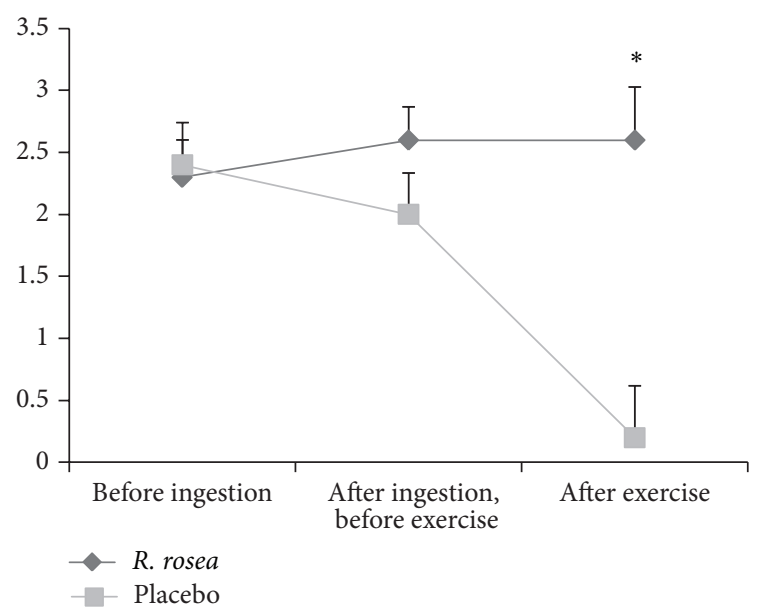

Figure 2: Mean \pm SE of perception of pleasure/displeasure before ingestion, after ingestion but before exercise, and after exercise between $R$. rosea and placebo conditions ( ${ }^{*} P=0.003$ ).

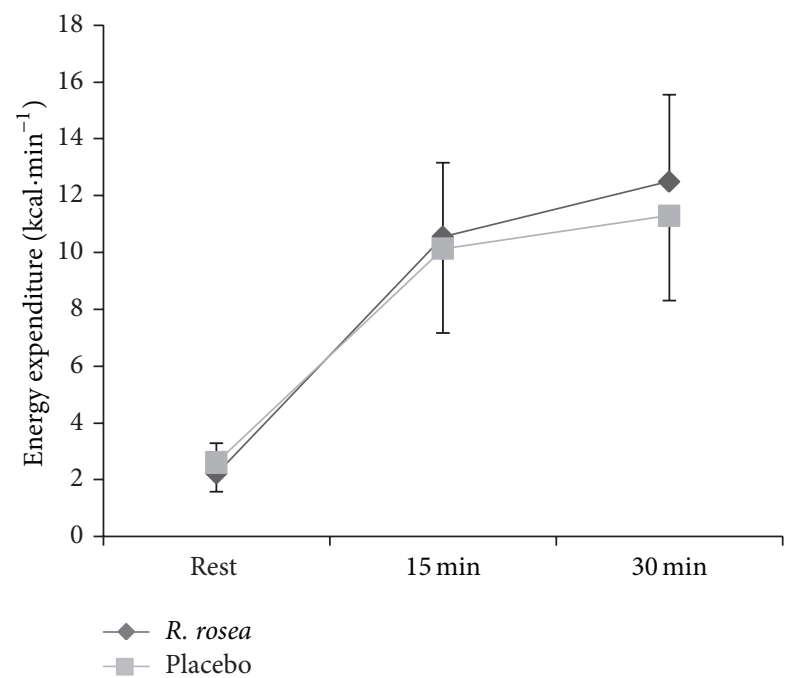

FIGURE 3: Mean \pm S.D. energy expenditure $\left(\mathrm{Kcal} \cdot \mathrm{min}^{-1}\right)$ during 30 min submaximal cycling between $R$. rosea and placebo conditions.

constant throughout exercise but was higher compared with the rest $\left(F 2,18=13.902, P<0.001, P \eta^{2}=0.770\right)$. The rate of fat oxidation (Figure 5) was higher than that at rest during exercise $\left(F 1,11=25.131, P<0.001, P \eta^{2}=0.736\right)$ but there was no significant main effect due to the substances ingested ( $\left.F 1,9=0.950, P=0.355, P \eta^{2}=0.095\right)$.

\section{Discussion}

Acute $R$. rosea ingestion has been reported to improve exercise performance $[13,14]$ in performance based tasks. Authors have suggested that this performance enhancement may result from $R$. rosea's action as an opioid producer $[2,13]$. However, use of performance based exercise tasks (e.g., time trial) in prior studies makes it difficult to assess the effect

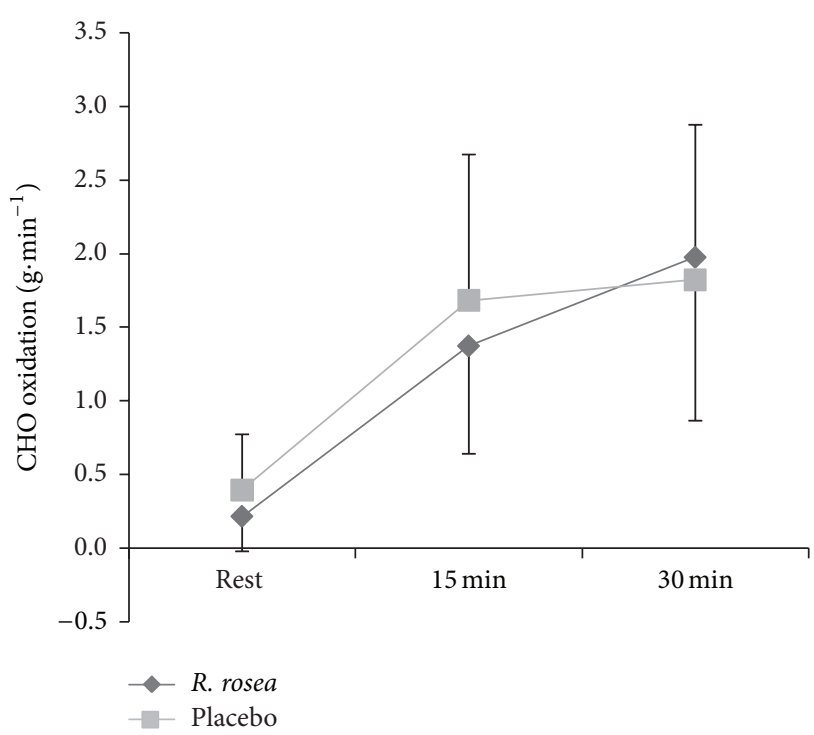

FIGURE 4: Mean \pm S.D. total CHO oxidation rate $\left(\mathrm{g} \cdot \mathrm{min}^{-1}\right)$ during 30 min submaximal cycling between $R$. rosea and placebo conditions.

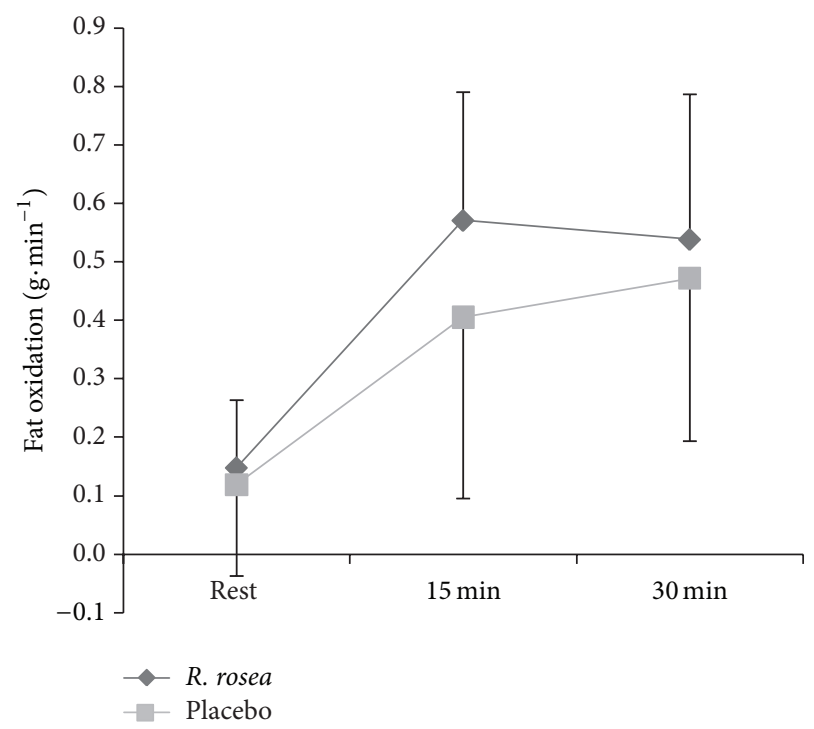

FIGURE 5: Mean \pm S.D. fat oxidation rate $\left(\mathrm{g} \cdot \mathrm{min}^{-1}\right)$ during $30 \mathrm{~min}$ submaximal cycling between $R$. rosea and placebo conditions.

of the substance ingested apart from the exercise intensity in tasks where intensity will fluctuate. Arguably, the sue of a constant load exercise protocol for 30 minutes is a more externally valid form of exercise for most individuals as it mirrors the minimum exercise recommendations for healthy adults [30]. No study to date has assessed the effects of $R$. rosea ingestion on mood, perceptions of exertion, arousal, and feelings of pleasure/displeasure during steady state exercise. In the current study, RPE and affect were measured prior to, during, and after 30-minute submaximal cycling at a moderate exercise intensity after ingestion of $3 \mathrm{mg} \cdot \mathrm{kg}^{-1} R$. rosea of placebo. This study is novel in that results demonstrated 
dampened RPE, greater vigor, perception of arousal, and pleasure in the presence of $R$. rosea compared to placebo. In the case of RPE and feelings of pleasure, these changes were seen at the end of the $30 \mathrm{~min}$ exercise bout. Thus, this data indicate that acute $R$. rosea ingestion positively modifies affect during relatively brief, steady state exercise at moderate intensity.

Previous studies have speculated that the ingestion of $R$. rosea can improve exercise performance via altered energy metabolism [16], activated by the synthesis or resynthesis of ATP in mitochondria and stimulated restorative energy processes after intense exercise [15]. However, the results of the present study suggest that altered substrate utilization is not responsible for the improved performance. The discrepancy between the findings of the present study in this respect and those of prior work $[15,16]$ may be due to varying preexercise fed state across studies. In the present study the Frayn [38] equation was employed to assess fat oxidation as this is the most widely applied equation for fat oxidation available. However, we acknowledge that other equations are also available [39] and may be preferable in future work. It is also important to note that there is no gold standard to validate whole body substrate oxidation but that assessment of substrate oxidation using indirect calorimetry may be prone to large errors [40]. Therefore, the acute increase in endogenous opioid production may play a role in enhancing exercise performance in the presence of $R$. rosea and is evidenced through changes in perceived exertion and exercise affect rather than changes in physiological markers.

The present study does have some limitations. The task used employed a bout of steady state exercise at a moderate intensity. Prior research has tended to employ protocols involving time trial performance [14] or time to exhaustion [13], neither of which is applicable to the typical exercising public. While the protocol employed in the present study may be valid when examining steady state exercise at a moderate intensity, it also might not fully address the typical training session undertaken by many recreational exercisers and athletes. However, given the lack of studies examining the effect of $R$. rosea on exercise performance such steady state protocols are useful in developing the extant literature in this area. We do acknowledge that by controlling exercise intensity based on a percentage of $\mathrm{V}_{2 \text { max }}$ we are unable to determine if participants were exercising below, at, or above their lactate threshold. Exercise across these different intensities may however change affective responses [22]. This should be considered in future studies. Future research might subsequently benefit from using a standardised protocol to mimic the type of exercise session commonly undertaken in gyms (e.g., combined aerobic and resistance exercise) in order to determine if $R$. rosea ingestion is practically useful in a more ecologically valid exercise task. The current study also examined participants who were recreationally active but were not specifically cycling trained. It has been suggested that less fit individuals may experience greater fatigue and discomfort during exercise which may reduce feelings of pleasure compared to more highly trained individuals. It may therefore be useful to compare the responses of participants of different training status in order to make more conclusive statements regarding the effect of $R$. rosea on variables such as perception of exertion, arousal, and pleasure. Furthermore, a posteriori power calculations indicated a sample size of 28 would have been required to detect a difference of $P=$ 0.05 at $95 \%$ power. However, due to the paucity of data on effects of $R$. rosea on exercise performance this study was exploratory in nature and subsequently used a relatively small number of participants. The current study may therefore be underpowered. Finally, the assessment of affect immediately on completion of the cycling bout might have resulted in elevated scores for feeling states due to the cessation of exercise as has been suggested previously [17]. It may be that the trajectory of pleasure and displeasure during and after exercise exhibits two distinct phases [22]. The first phase involves a decline or increase of affective responses during exercise, whereas the second phase involves an improvement or rebound of affective responses after exercise. As measures of affect were only taken on completion of the exercise bouts, the data presented here are only representative of the rebound phase of exercise in the presence of $R$. rosea and placebo. Future research should therefore attempt to assess affect during exercise in addition to immediately on cessation in order to more effectively capture the time course of affective responses to exercise following ingestion of different substances.

\section{Conclusions}

Ingestion of $R$. rosea favourably influenced RPE and exercise affect without changes in energy expenditure or substrate utilization during 30 minutes of submaximal cycling performance at moderate intensity in regularly active adults. These changes support the efficacy of acute $R$. rosea ingestion in positively enhancing psychophysiological responses to submaximal exercise performance.

\section{Conflict of Interests}

The authors declare that there is no conflict of interests regarding the publication of this paper.

\section{References}

[1] T. B. Walker and R. A. Robergs, "Does Rhodiola rosea possess ergogenic properties?” International Journal of Sport Nutrition and Exercise Metabolism, vol. 16, no. 3, pp. 305-315, 2006.

[2] R. P. Brown, P. L. Gerbarg, and Z. Ramazanov, "Rhodiola rosea: a phytomedicinal overview," HerbalGram, vol. 56, pp. 40-52, 2002.

[3] M. Abidov, S. Grachev, R. D. Seifulla, and T. N. Ziegenfuss, "Extract of Rhodiola rosea radix reduces the level of Creactive protein and creatinine kinase in the blood," Bulletin of Experimental Biology and Medicine, vol. 138, no. 1, pp. 63-64, 2004.

[4] A. Skarpanska-Stejnborn, L. Pilaczynska-Szczesniak, P. Basta, and E. Deskur-Smielecka, "The influence of supplementation with Rhodiola rosea L. extract on Selected redox parameters in professional rowers," International Journal of Sport Nutrition and Exercise Metabolism, vol. 19, no. 2, pp. 186-199, 2009. 
[5] V. A. Shevtsov, B. I. Zholus, V. I. Shervarly et al., "A randomized trial of two different doses of a SHR-5 Rhodiola rosea extract versus placebo and control of capacity for mental work," Phytomedicine, vol. 10, no. 2-3, pp. 95-105, 2003.

[6] A. A. Spasov, G. K. Wikman, V. B. Mandrikov, I. A. Mironova, and V. V. Neumoin, "A double-blind, placebo-controlled pilot study of the stimulating and adaptogenic effect of Rhodiola rosea SHR-5 extract on the fatigue of students caused by stress during an examination period with a repeated low-dose regimen," Phytomedicine, vol. 7, no. 2, pp. 85-89, 2000.

[7] V. Darbinyan, A. Kteyan, A. Panossian, E. Gabrielian, G. Wikman, and H. Wagner, "Rhodiola rosea in stress induced fatigue-a double blind cross-over study of a standardized extract SHR-5 with a repeated low-dose regimen on the mental performance of healthy physicians during night duty," Phytomedicine, vol. 7, no. 5, pp. 365-371, 2000.

[8] L. Mattioli, C. Funari, and M. Perfumi, "Effects of Rhodiola rosea L. extract on behavioural and physiological alterations induced by chronic mild stress in female rats," Journal of Psychopharmacology, vol. 23, no. 2, pp. 130-142, 2009.

[9] F. T. Lee, T. Y. Kuo, S. Y. Liou, and C. T. Chien, "Chronic Rhodiola rosea extract supplementation enforces exhaustive swimming tolerance," The American Journal of Chinese Medicine, vol. 37, no. 3, pp. 557-572, 2009.

[10] T. B. Walker, S. A. Altobelli, A. Caprihan, and R. A. Robergs, "Failure of Rhodiola rosea to alter skeletal muscle phosphate kinetics in trained men," Metabolism: Clinical and Experimental, vol. 56, no. 8, pp. 1111-1117, 2007.

[11] S. N. Colson, F. B. Wyatt, D. L. Johnston, L. D. Autrey, Y. L. FitzGerald, and C. P. Earnest, "Cordyceps sinensis- and Rhodiola rosea-based supplementation in male cyclists and its effect on muscle tissue oxygen saturation," Journal of Strength and Conditioning Research, vol. 19, no. 2, pp. 358-363, 2005.

[12] C. P. Earnest, G. M. Morss, F. Wyatt et al., "Effects of a commercial herbal-based formula on exercise performance in cyclists," Medicine and Science in Sports and Exercise, vol. 36, no. 3, pp. 504-509, 2004.

[13] K. de Bock, B. O. Eijnde, M. Ramaekers, and P. Hespel, "Acute Rhodiola rosea intake can improve endurance exercise performance," International Journal of Sport Nutrition and Exercise Metabolism, vol. 14, no. 3, pp. 298-307, 2004.

[14] E. E. Noreen, J. G. Buckley, S. L. Lewis, J. Brandauer, and K. J. Stuempfle, "The effects of an acute does of Rhodiola rosea on endurance exercise performance," Journal of Strength and Conditioning Research, vol. 27, no. 3, pp. 839-847, 2013.

[15] M. Abidov, F. Crendal, S. Grachev, R. Seifulla, and T. Ziegenfuss, "Effect of extracts from Rhodiola rosea and Rhodiola crenulata (Crassulaceae) roots on ATP content in mitochondria of skeletal muscles," Bulletin of Experimental Biology and Medicine, vol. 136, no. 6, pp. 585-587, 2003.

[16] M. Perfumi and L. Mattioli, "Adaptogenic and central nervous system effects of single doses of $3 \%$ rosavin and $1 \%$ salidroside Rhodiola rosea L. extract in mice," Phytotherapy Research, vol. 21, no. 1, pp. 37-43, 2007.

[17] P. Ekkekakis, G. Parfitt, and S. J. Petruzzello, “The pleasure and displeasure people feel when they exercise at different intensities: decennial update and progress towards a tripartite rationale for exercise intensity prescription," Sports Medicine, vol. 41, no. 8, pp. 641-671, 2011.

[18] M. J. Duncan and S. W. Oxford, "The effect of caffeine ingestion on mood state and bench press performance to failure," Journal of Strength and Conditioning Research, vol. 25, no. 1, pp. 178-185, 2011.

[19] T. A. Astorino, T. Cottrell, A. T. Lozano, K. Aburto-Pratt, and J. Duhon, "Effect of caffeine on RPE and perceptions of pain, arousal, and pleasure/displeasure during a cycling time trial in endurance trained and active men," Physiology and Behavior, vol. 106, no. 2, pp. 211-217, 2012.

[20] S. H. Backhouse, N. C. Bishop, S. J. H. Biddle, and C. Williams, "Effect of carbohydrate and prolonged exercise on affect and perceived exertion," Medicine and Science in Sports and Exercise, vol. 37, no. 10, pp. 1768-1773, 2005.

[21] D. Kahneman, B. L. Fredrickson, C. A. Schreiber, and D. A. Redelmeier, "When more pain is preferred to less: adding a better end," Psychological Science, vol. 4, no. 6, pp. 401-405, 1993.

[22] P. Ekkekakis, E. E. Hall, and S. J. Petruzzello, "Variation and homogeneity in affective responses to physical activity of varying intensities: an alternative perspective on dose-response based on evolutionary considerations," Journal of Sports Sciences, vol. 23, no. 5, pp. 477-500, 2005.

[23] K. A. Barbour, T. M. Edenfield, and J. A. Blumenthal, "Exercise as a treatment for depression and other psychiatric disorders: a review," Journal of Cardiopulmonary Rehabilitation and Prevention, vol. 27, no. 6, pp. 359-367, 2007.

[24] M. D. Hoffman and D. R. Hoffman, "Exercisers achieve greater acute exercise-induced mood enhancement than nonexercisers," Archives of Physical Medicine and Rehabilitation, vol. 89, no. 2, pp. 358-363, 2008.

[25] E. O. Acevedo, D. L. Gill, A. H. Goldfarb, and B. T. Boyer, "Affect and perceived exertion during a two-hour run," International Journal of Sport Psychology, vol. 27, no. 3, pp. 286-292, 1996.

[26] S. G. DaSilva, L. Guidetti, C. F. Buzzachera et al., “The influence of adiposity on physiological, perceptual, and affective responses during walking at a self-selected pace," Perceptual and Motor Skills, vol. 109, no. 1, pp. 41-60, 2009.

[27] L. Moseley and A. E. Jeukendrup, "The reliability of cycling efficiency," Medicine and Science in Sports and Exercise, vol. 33, no. 4, pp. 621-627, 2001.

[28] G. Borg, "Perceived exertion as an indicator of somatic stress," Scandinavian Journal of Rehabilitation Medicine, vol. 2, no. 2, pp. 92-98, 1970.

[29] A. W. Midgley, L. R. McNaughton, R. Polman, and D. Marchant, "Criteria for determination of maximal oxygen uptake: a brief critique and recommendations for future research," Sports Medicine, vol. 37, no. 12, pp. 1019-1028, 2007.

[30] American College of Sports Medicine, ACSM's Guidelines for Exercise Testing and Prescription, Lippincott Williams \& Wilkins, Baltimore, Md, USA, 2013.

[31] N. Guo, Z. Hu, X. Fan et al., "Simultaneous determination of Salidroside and its aglycone metabolite p-tyrosol in rat plasma by liquid chromatography-tandem mass spectrometry," Molecules, vol. 17, no. 4, pp. 4733-4754, 2012.

[32] R. J. Robertson, Perceived Exertion for Practitioners, Human Kinetics, Champaign, Ill, USA, 2004.

[33] C. J. Hardy and W. J. Rejeski, "Not what, but how one feels: the measurement of affect during exercise," Journal of Sport and Exercise Psychology, vol. 11, pp. 304-317, 1989.

[34] S. Svebak and S. Murgatroyd, "Metamotivational dominance. A multimethod validation of reversal theory constructs," Journal of Personality and Social Psychology, vol. 48, no. 1, pp. 107-116, 1985. 
[35] P. Terry and A. Lane, User Guide for the Brunel Mood State Inventory (BRUMS), University of Southern Australia, Queensland, Australia, 2003.

[36] P. C. Terry and A. M. Lane, "Normative values for the profile of mood states for use with athletic samples," Journal of Applied Sport Psychology, vol. 12, no. 1, pp. 69-85, 2000.

[37] J. B. Weir, "New methods for calculating metabolic rate with special reference to protein metabolism," Journal of Physiology, vol. 109, no. 1-2, pp. 1-9, 1949.

[38] K. N. Frayn, "Calculation of substrate oxidation rates in vivo from gaseous exchange," Journal of Applied Physiology Respiratory Environmental and Exercise Physiology, vol. 55, no. 2, pp. 628-634, 1983.

[39] A. E. Jeukendrup and G. A. Wallis, "Measurement of substrate oxidation during exercise by means of gas exchange measurements," International Journal of Sports Medicine, vol. 26, supplement 1, pp. S28-S37, 2005.

[40] Y. Schutz, "On problems of calculating energy expenditure and substrate utilization from respiratory exchange data," European Journal of Nutrition, vol. 36, no. 4, pp. 255-262, 1997. 


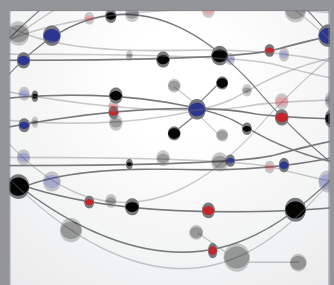

The Scientific World Journal
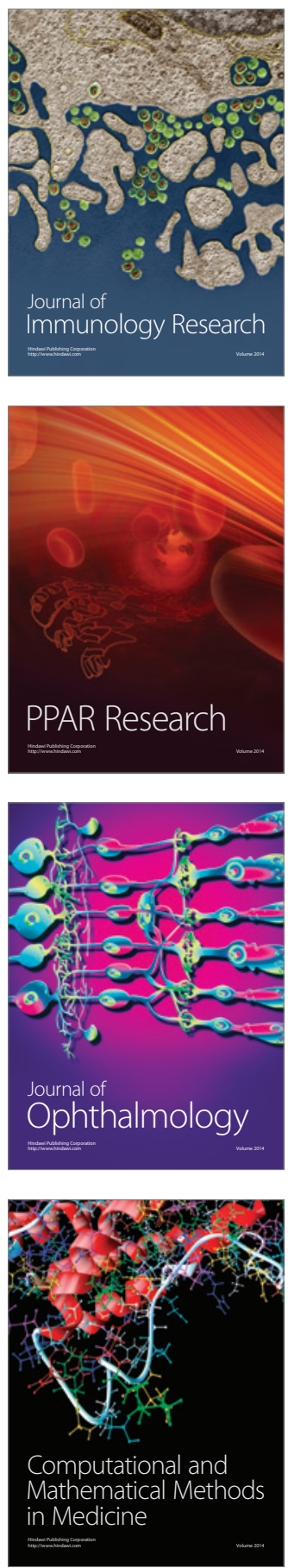

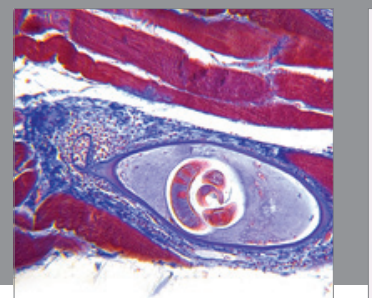

Gastroenterology

Research and Practice
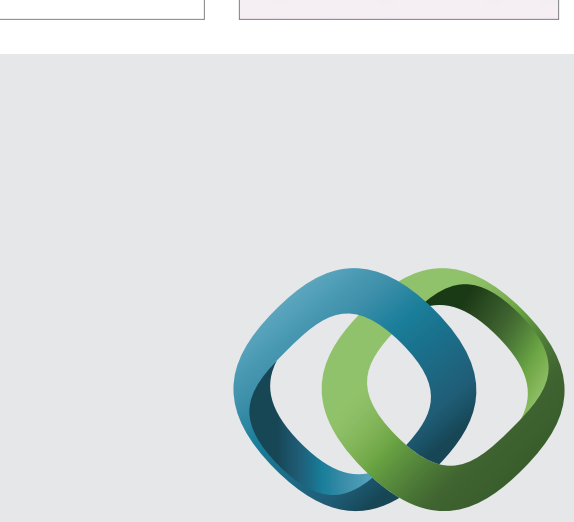

\section{Hindawi}

Submit your manuscripts at

http://www.hindawi.com
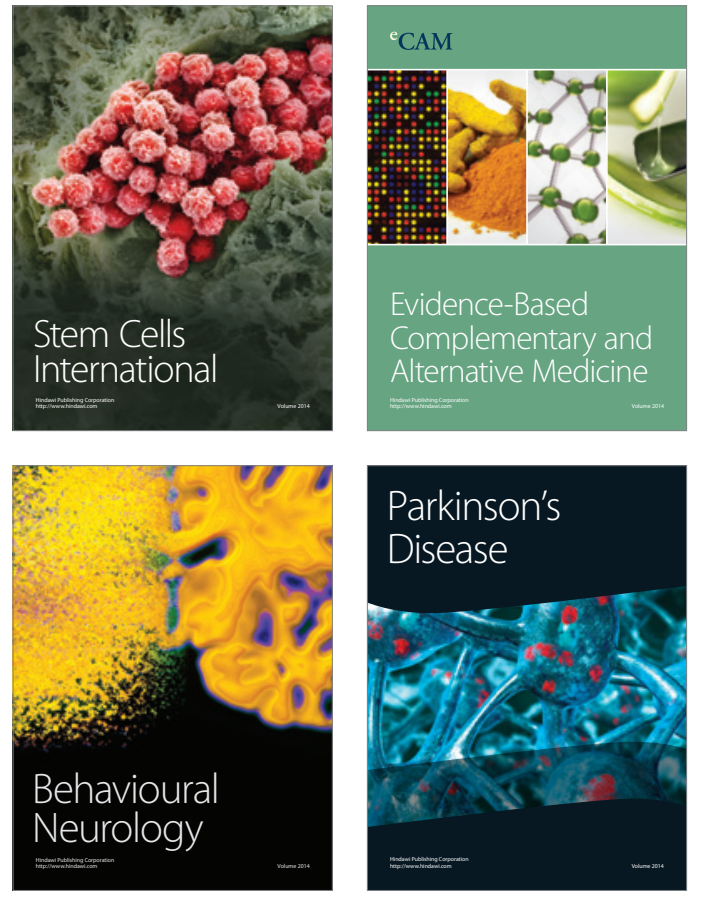
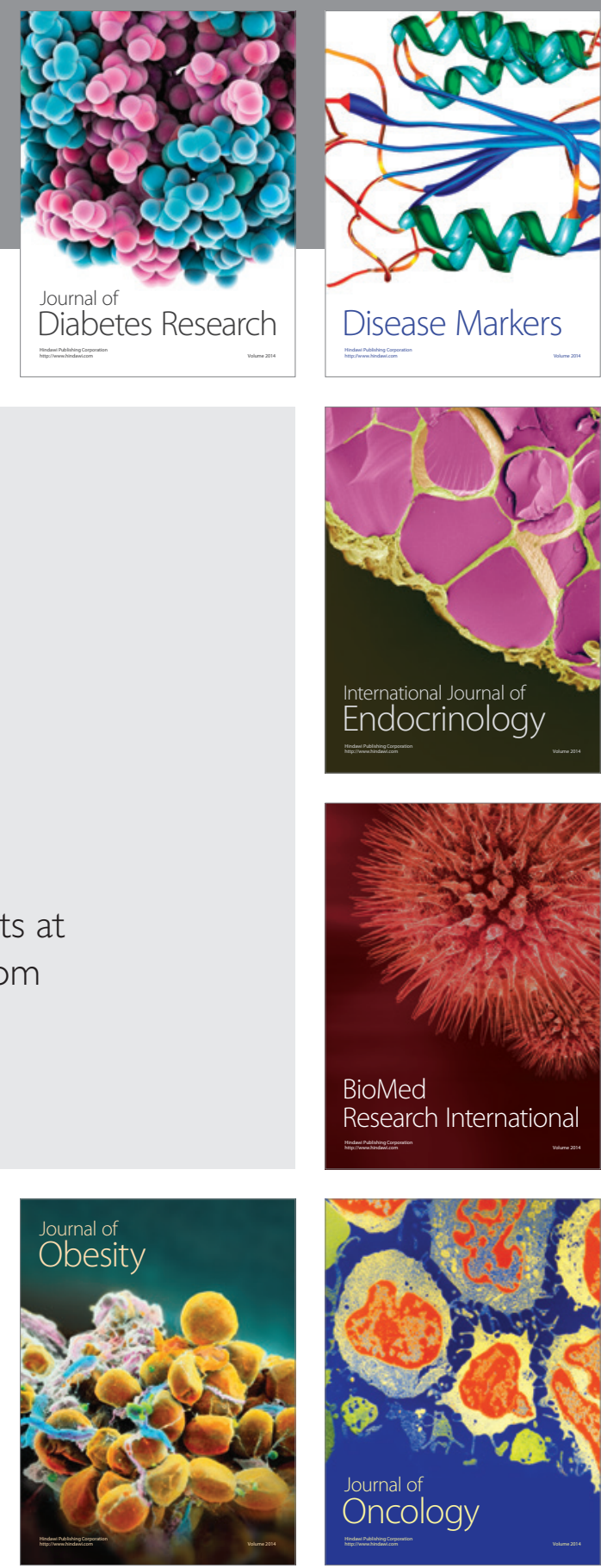

Disease Markers
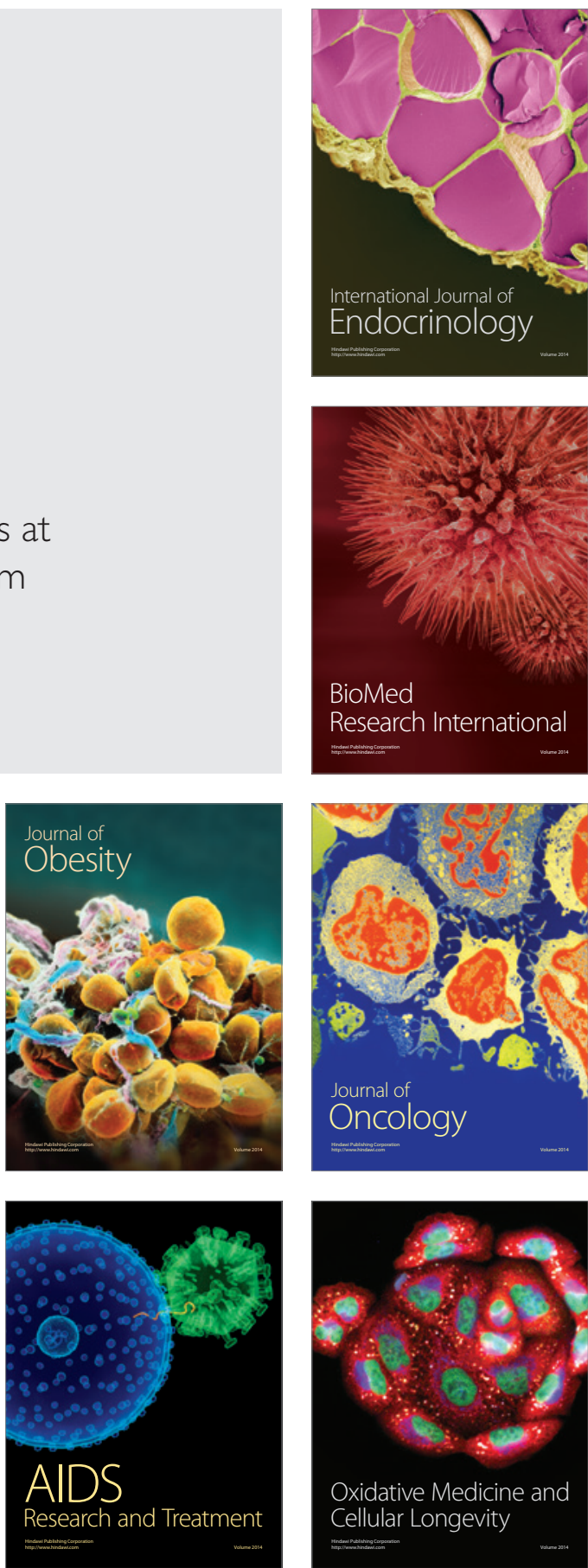